\title{
A C A S E
}

IN WHICH

\section{ABDOMINAL SECTION WAS SUCCESSFULLY PERFORMED FOR INTUSSUSCEPTION IN AN INFANT SEVEN MONTHS OLD.}

\author{
BY \\ HOWARD MARSH, F.R.C.S., \\ ASSISTANT-SURGEON TO ST. BARTHOLOMEW'S HOSPITAL AND TO THE \\ HOSPITAL FOR SICK OHILDREN.
}

(Received August 16th - Read December 14th, 1875.)

ON the 11th of last April, while staying a night in the neighbourhood, I was asked by Dr. Miller and Dr. Barnes, of Eye, in Suffolk, to see a male infant seven months old, with intussusception. Dr. Barnes told me that on March 29th-fourteen days previously-the child had been seized with diarrhœa, sickness, and occasional griping pains in the abdomen. These symptoms, none of which were severe, and which seemed due to an attack of ordinary catarrhal enteritis, were at once relieved by small doses of castor oil emulsion, and natural feculent evacuations were passed. In two or three days, however, the griping and sickness returned, now accompanied with tenesmus; and the motions were observed to contain a considerable quantity of slimy mucus, mixed with blood. The child remained in much the same condition, and without any 
marked disturbance of his general health until twelve hours before the consultation, when the whole complexion of his illness suddenly changed. His abdominal pain became very severe, sickness was frequent and tenesmus violent, and almost constant; and he grew pale and very restless.

Having been sent for, Dr. Barnes found a portion of bowel projecting two inches beyond the anus, with the ileo-cæcal valve clearly seen at the extremity of the protrusion; while, in the abdomen, a firm, cylindrical tumour could be felt, extending from the umbilicus to the left iliac fossa. A careful trial both with insufflation, and the injection of warm water into the large intestine, while the child was under chloroform, failed to reduce the invagination.

On reaching the house I found Dr. Barnes's account of the case had been so complete that there was nothing to be added to it. The intussusception was now lying just within the sphincter; but, when the child strained, it was protruded more than two inches beyond the anus. The mucous membrane was of a deep plum colour from congestion, and looked œdematous, but it was bright and glossy, and presented no abrasion of its surface. Almost at the extremity of the volvulus, but rather at the side, the ileo-cæcal opening, bordered by the two nearly horizontal cusps of the valve, was distinctly seen. The child's napkin was stained with serum and blood, which oozed in considerable quantity from the mucous membrane of the invaginated bowel. In the abdomen a firm, sausageshaped tumour could be very readily felt; indeed it could be plainly seen when the child, in straining, tightened his muscles. Its upper end, abrupt and well-defined, was placed just to the left of the umbilicus; and from this point its length could be traced in the course of the descending colon and rectum down into the lower part of the pelvis. The child lay in his nurse's arms retching every two or three minutes; with a pale face, sunken halfclosed eyes, a small and very rapid pulse, and shallow, 
hurried respiration. The parents begged that whatever gave a reasonable hope of saving his life might be done.

When he was under chloroform the abdomen was opened by an incision about two inches long, beginning in the middle line, immediately below the umbilicus, and running downwards towards the pubes. It was found that when the recti had been pulled aside the subjacent tissues, including the peritoneum, were so thin that mach care was needed to avoid injuring the intestine, which bulged forward into the wound. Two fingers were now passed down to the upper end of the intussusception, the position of which was already precisely known, and an effort was made to draw out the intestine. This quite failed, and the impression left on my mind at the time was that such an attempt would be very little likely to succeed, except, perhaps, in the case of a very short intussusception; for with only two fingers it was impossible to fix the tumour, and, at the same time, to pull out the volvulus. I therefore brought the upper end out through the wound, and gently dragged at the entering portion of the gut. For a moment it would not come, but, using a slightly increased force, I felt that its distal end was started, and then it glided smoothly up the colon, the canal of which seemed large enough to let it pass along with the utmost facility. At last the cæcum and its appendix emerged, their serous surface being clearly presented to view. What length of intestine had been invaginated cannot be accurately stated, but obviously this included at least half the colon and an equal portion of the small intestine. When the intussusception had been reduced, a considerable proportion of the intestines, with a part of the great omentum, lay on the surface of the abdomen; and the return of these coils through an opening even two inches long proved to be by far the most difficult part of the operation ; but with Dr. Barnes' help, and while Dr. Miller kept the child still under chloroform, this was accomplished without much delay. The wound was then closed with two harelip pins and voL. LIX. 
some superficial sutures, and supported with long pieces of strapping and a bandage. There was no bleeding of any importance during the operation. The case was subsequently entirely under the care of Dr. Miller and Dr. Barnes. Small doses of laudanum were given three or four times in the course of the next twenty-four hours; and the child drank freely of milk and water, to which, when there seemed occasion, a few drops of brandy were added. The sickness ceased immediately after the operation, and did not return; flatus and a small quantity of blood-stained mucus were passed from the bowel twice or three times in the first twelve hours; the child had no pain, and the abdomen remained soft and free from tenderness.

On the 13th Dr. Barnes found the child bright and playing; without pain or fever, and with a pulse of 95 .

On the 14th he had slept almost all night. A feculent motion was passed early in the day.

On the 15th the pins were removed, eighty-four hours after the operation; the wound was found nearly healed, and its edges showed no tendency to separate. From this time there was nothing to report except that, in a few days, the child had perfectly recovered. He is still in good health.

Remarks.-The chief features of interest in this case will be placed in the strongest light if, instead of discussing them alone, they are examined in their bearing on Mr. Hutchinson's paper on intussusception, published in the 57th volume of the Society's 'Transactions.' Before that communication was read it was the very general opinion that abdominal section ought not to be performed in cases of intussusception, because, among other reasons, the intestine became so damaged by inflammation and strangulation, and so fixed by the mutual adhesion of its different layers, that it could not be safely drawn out. But Mr. Hutchinson directed particular attention to the fact-which, though it had been often noticed before at post-mortem examinations, had never been credited with 
much clinical importance-that, in many cases, the condition of an intussusception is not that of inflammation, strangulation, or adhesion of its layers, but of simple invagination; the volvulus lying loose in its sheath, in a state comparable with that of a portion of intestine prolapsed into a hernial sac; but through which the circulation is still free, and surrounding which there are no adhesions. The case just related bears out this observation in a striking manner. The child's illness was divided into two distinct periods; the first-extending to the early part of the fourteenth day-in which all the symptoms were so chronic that they raised no suspicion as to the real nature of the affection; the second-occupying the twelve hours immediately preceding the operation -in which all the symptoms were very acute. Doubtless the onset of these acute symptoms marked the time at which mere invagination was succeeded by inflammation and strangulation. And what was found at the operation corresponded with this view. No firm adhesions were discovered; but in drawing out the intestine just so much resistance was at first encountered as might be due to œdema, and commencing adhesions, resulting from twelve hours of acute strangulation. Difficult or even impossible as it may be in, perhaps, the majority of cases, to feel any certainty as to the condition of the intestine, it seemed in this instance safe to conclude that it had not been materially damaged.

Mr. Hutchinson refers to the effect which the age of the patient is likely to have on the result of the operation; and he remarks that " in the absence of any data as to the manner in which operations of this kind are borne by very young children, we shall probably be right in believing that they are far less hopeful than in those that are somewhat older." The evidence derived from this case, however, points the other way, for though the patient was only seven months old, though he was in a condition of collapse at the time of the operation, though the intussusception was very extensive, and though a con- 
siderable length of the intestine lay at one time on the surface of the abdomen and was not returned without free handling, yet recovery took place without a bad symptom, and with no more trouble or suffering than usually attends an operation for harelip; and the patient, Dr. Miller and Dr. Barnes informed me, was convalescent on the fourth day.

In recording this case I must beg permission of the Society to add that I am far from advocating that abdominal section should be performed indiscriminately in cases of intussusception. Such a course would inevitably tend to bring surgery into disrepute. But that there are instances in which the operation should be done is proved by Mr. Hutchinson's case and by that related above.

If the diagnosis is certain, and if other means, carefully tried, have failed, my conviction is that the operation ought at once to be performed; first, in cases in which strangulation is acute and quite recent; that is, of not more than twelve or, at the most, eighteeen hours' duration. Secondly, in cases which are chronic, and in which there have been no symptoms of inflammation or strangulation. The responsibility of operating must always be great and failures must be expected; but I believe the responsibility of withholding the operation in all cases will be greater still. 